MS07-P09

\section{Structural insights into the dimeric human PNPase revealing why the disease-linked mutants exhibit lower RNA import and degradation activities}

Bagher Golzarroshan ${ }^{1}$, Hanna Yuan ${ }^{1}$

1. Institute of molecular biology, Taipei, Taiwan

email: golzar1984@gate.sinica.edu.tw

Polynucleotide phosphorylase (PNPase) is an evolutionary conserved 3'-to-5' exoribonuclease that functions differently among species, ranging from RNA turnover in bacteria to RNA transport in mammals. In human, PNPase is essential for importing a subgroup of noncoding RNAs, including 5S rRNA, MRP RNA and RNase P RNA, into the mitochondria. Mutations in PNPase are thus linked to impair in the RNA import pathway and severe mitochondrial dysfunction and diseases. PNPase is primarily assembled into a trimeric conformation with a central channel for binding of a single-stranded RNA and leading its 3 ' end into the active site for degradation. In this study we show that the trimeric assembly of PNPase is affected by the disease-linked mutations, including Q378R and D475G. PNPase is oligomerized into a dimeric conformation after introducing the disease-linked mutations, and these PNPase mutants have lower RNA-binding and degrading activities as compared to the wild-type PNPase. Moreover, we found that $\mathrm{S} 1$ domain of PNPase is responsible for the interaction with the stem-loop motif of imported RNAs. We further determined the crystal structure of the dimeric form of the S1-truncated PNPase at a resolution of $2.8 \AA$. Combining with small angle X-ray scattering (SAXS), we showed that both of the RNA-binding $\mathrm{KH}$ and $\mathrm{S} 1$ domains are not fully accessible in the dimeric structure, explaining why these dimeric PNPase mutants interact with RNA poorly. Taken together these results show that mutations at the interface of the trimeric PNPase tend to produce a dimeric protein with obstructed RNA-binding surfaces, thus impairing both of its RNA import and degradation activities and leading to mitochondria disorders.

References:

Ameln, S. v., Wang, G., Boulouiz, R., Rutherford, M. A., Smith, G. M., Li, Y., Pogoda, H.-M., Nürnberg, G., Stiller, B., Volk, A. E., Borck, G., Hong, J. S., Goodyear, R. J., Abidi, O., Nürnberg, P., Hofmann, K., Richardson, G. P., Hammerschmidt, M., Moser, T., Wollnik, B., Koehler, C. M., Teitell, M. A., Barakat, A. \& Kubisch, C. (2012). Am. J. Human Genet. 91, 1-9.

Vedrenne, V., Gowher, A., De Lonlay, P., Nitschke, P., Serre, V., Boddaert, N., Altuzarra, C., Mager-Heckel, A. M., Chretien, F., Entelis, N., Munnich, A., Tarassov, I. \& Rotig, A. (2012). American Journal of Human Genetics 91, 912-918.

Wang, G., Chen, H. W., Oktay, Y., Zhang, J., Allen, E. L., Smith, G. M., Fan, K. C., Hong, J. S., French, S. W., McCaffery, J. M., Lightowlers, R. N., Morse, H. C., 3rd, Koehler, C. M. \& Teitell, M. A. (2010). Mol. Cell 142, 456-467.

Keywords: Crystal Structure, Diseases, Nucleic Acid
MS08- Membranes and membrane interacting proteins

Chairs: Prof. Susanna Törnroth-Horsefield, Prof.Adrian Goldman

MS08-P01

\section{Structure of an essential inner membrane protein-LPS complex}

Thomas Clairfeuille ${ }^{1}$, Kerry Buchholz ${ }^{2}$, Jian Payandeh ${ }^{1}$, Steven Rutherford ${ }^{2}$

1. Department of Structural Biology, Genentech, South San Francisco, United States of America

2. Department of Infectious diseases, Genentech, South San Francisco, United States of America

email: clairfet@gene.com

Multi-drug resistant Gram-negative bacteria (GNB) represent a global health threat and urgent unmet medical need. A defining feature of GNB is their outer membrane (OM), where lipopolysaccharide (LPS) establishes a permeability barrier that notoriously frustrates most antibiotic discovery efforts. LPS is also a potent stimulator of the innate immune system and responsible for a growing number of deaths, where $\sim 5$ million childhood deaths are attributed to bacterial sepsis every year. Despite the clear biomedical and pharmacological relevance of LPS, the essential mechanisms that coordinate LPS and phospholipid transport to the OM remain unknown. During efforts to identify new targets for the treatment of GNB, we became focused on a multipass integral inner membrane protein of unknown function that we call PlbA. Here, we find that PlbA is essential to establish infection in Escherichia coli, and that PlbA depletion produces defects in the OM barrier. High-resolution X-ray crystallographic structural analyses using traditional and XFEL diffraction methods of PlbA identifies a superfamily of transmembrane proteins that catalyze diverse modifications in the envelopes of Gram-positive and Gram-negative bacteria. However, the unique architecture of PlbA reveals a defunct active site. Instead, an LPS molecule is selectively coordinated to an unanticipated LPS-binding motif found along the periplasmic membrane leaflet of PlbA, where core-lipid A binding is achieved through an intricate network of backbone and water mediated interactions. Notably, this PlbA-LPS interaction complex is unprecedented when compared to known structures of selective LPS binding proteins, including the LPS transporter MsbA and the immune Toll-like receptor TLR4. Introduction of disruptive single point mutations at the PlbA-LPS interface leads to defects in the OM barrier. Overall, this work reveals unprecedented insight into the structural basis of LPS perception within the inner membrane of GNB and implicates PlbA as an LPS-sensor that helps coordinate LPS and PL transport to the OM.

Keywords: lipopolysaccharide, outer membrane, lipid transport 